\title{
Volume et temps de rétention du contenu abomasal chez le mouton nourri ad libitum
}

\author{
C Dardillat, R Baumont \\ INRA, unité Ingestion, station de recherches sur la Nutrition des herbivores, centre de recherches \\ de Clermont-Ferrand/Theix, 63122 Saint-Genès-Champanelle, France
}

\begin{abstract}
Summary - Volume and retention time of abomasal content in sheep fed ad libitum. Three sheep fitted a large retrosternal abomasal cannula were fed ad libitum once per day a diet of orchard grass or lucerne hay or straw with or without barbey. Volume and retention time of abomasal content were estimated by Cr-EDTA. Retention time $(r=-0.94)$ and abomasal outflow $(r=0.78)$ but not abomasal volume were closely correlated with the level of intake.
\end{abstract}

Bien que le débit duodénal soit peu différent en moyenne du débit ruminal (Moir, 1984), on ignore dans quelle mesure les variations intrajournalières de celui-ci (Dardillat, 1987) peuvent être tamponnées par les variations spontanées du volume et du taux de matière sèche du contenu abomasal et, inversement, si les variations de débit duodénal traduisent des variations de la vidange ruminale. Pour apprécier ce rôle tampon de l'abomasum, nous avons étudié chez le mouton les variations du volume du contenu abomasal (VCA), du temps de rétention moyen de la phase liquide (TRM), et de la teneur en matière sèche (MS) au cours de la journée en fonction de la nature de la ration.

Matériel et méthodes - Trois moutons Texel ont été munis, plus d'un mois avant les mesures, d'une canule abomasale rétrosternale en polyester stratifié de diamètre intérieur et extérieur de 25 et $26,8 \mathrm{~mm}$, étanche grâce à une collerette en dacron colonisable. lis ont été alimentés 1 fois/j ad libitum, successivement avec un foin de dactyle, un foin de luzerne, une paille (66\%) complétée par de la farine d'orge $(22,5 \%)$ et du tourteau de soja $(11,5 \%)$, et enfin une paille seule. Le volume gastrique avant et au cours des $7 \mathrm{~h}$ suivant le repas a été mesuré par la dilution instantanée d'un marqueur de la phase liquide (Cr-EDTA) injecté dans la caillette et dispersé par 20 aspirations-réinjections rapides de $100 \mathrm{ml}$ de contenu à la seringue. Entre
2 mesures de volume, le TRM a été calculé après régression linéaire de la croissance des logarithmes de la concentration en marqueur mesurée aux temps $0,10,20,30,60,70$ et 80 $\mathrm{min}$. Pour chaque animal et chaque régime, au moins 8 mesures de VCA et 4 mesures de TRM ont été réalisées avant le repas, $2 h$ et $5 h$ après. Quatre mesures de VCA et 2 de TRM étaient réalisées par animal chaque jour expérimental.

Résultats et discussion - La décroissance en fonction du temps du logarithme des concentrations en marqueur est toujours linéaire ( $r$ compris entre 0,95 et 0,99 ) et passe par la concentration initiale mesurée, confirmant ainsi la validité de la mesure du VCA par dilution instantanée. Aucun des paramètres mesurés ne varie en fonction de l'intervalle de temps qui sépare l'heure de prélèvement de la distribution du repas. Par contre, la teneur en MS et le TRM sont significativement affectés par la nature de la ration. Ils augmentent pour les rations de dactyle et de luzerne (tableau I). II existe une corrélation négative étroite entre le TRM et la quantité ingérée quel que soit le mode de calcul (moyenne ou données individuelles intrarégime ou tous régimes confondus). La corrélation entre les débits duodénaux et la quantité ingérée, elle aussi significative, est moins étroite (tableau II). 
En conclusion il est remarquable de constater que le TRM dans l'abomasum diminue quand le niveau d'ingestion augmente, sans que le VCA ne soit modifié. La réplétion gastrique n'est pas la cause des variations de débit duodénal. Les variations du VCA qui ne sont liées à aucun des facteurs mesurés (ingestion, régime, vitesse de transit) peuvent être la conséquence des variations cycliques de débit duodénal liées aux complexes myoélectri- ques moteurs (Malbert et Baumont, 1989). L'effet tampon du réservoir abomasal serait donc peu important vis-à-vis des variations de débit à la sortie du rumen.

Dardillat C (1987) Rèprod Nutr Dév 27, 231-232

Malbert $\mathrm{CH}$, Baumont $\mathrm{R}$ (1989) Br J Nutr 61, 699-714

Moir RJ (1984) Ruminant physiology concepts and consequences (SK Baker, JM Gawthorne, JB Mackintosh, DB Pursen, eds) 85-92

Tableau I. Influence de la nature de la ration sur la quantité ingérée et les caractéristiques du contenu abomasal chez 3 moutons nourris ad libitum.

\begin{tabular}{|c|c|c|c|c|c|c|}
\hline & Dactyle & Luzerne & $\begin{array}{c}\text { Paille } \\
+ \text { concentré }\end{array}$ & Paille & $E T R$ & $\begin{array}{l}\text { Corrélation } \\
\text { avec QI }\end{array}$ \\
\hline Quantité ingérée (g MS/j) & $1534^{a}$ & $1497^{a}$ & $1183^{a b}$ & $736^{\mathrm{b}}$ & 234 & \\
\hline Volume abomasal (ml) & $583^{a}$ & $509^{a}$ & $551^{a}$ & $622^{a}$ & 125 & $-0,07^{N S}$ \\
\hline Teneur en MS (\%) & $6,21^{a}$ & $6,95^{\mathrm{ab}}$ & $8,24^{b c}$ & $8,71^{c}$ & 0,61 & $-0,59^{N S}$ \\
\hline Contenu sec (g de MS) & $34,9^{a}$ & $35,5^{a}$ & $44,7^{a}$ & $54,7^{a}$ & 9,5 & $-0,55^{\mathrm{NS}}$ \\
\hline TRM du liquide (min) & $58,4^{a}$ & $59,3^{a}$ & $88,3^{a b}$ & $110,5^{b}$ & 17,9 & $-0,94^{* \star}$ \\
\hline Débit liquide (ml/min) & $10,73^{a}$ & $8,66^{a}$ & $6,34^{a}$ & $5,75^{a}$ & 2,93 & $0,78^{* *}$ \\
\hline Débit MS (g MS/h) & $38,3^{a}$ & $35,9^{a}$ & $31,4^{\mathrm{a}}$ & $30,5^{\mathrm{a}}$ & 10,0 & $0,72^{*}$ \\
\hline
\end{tabular}

ETR : écart résiduel; ${ }^{a}, \mathrm{~b}, c$ : sur une mêrme ligne, les moyennes qui n'ont aucun exposant commun diffèrent ( $P<$ $0,05)$; NS: non significatif; ${ }^{\circ}: P<0,05 ;{ }^{*}: P<0,01$. TRM : temps de rétention moyen. 